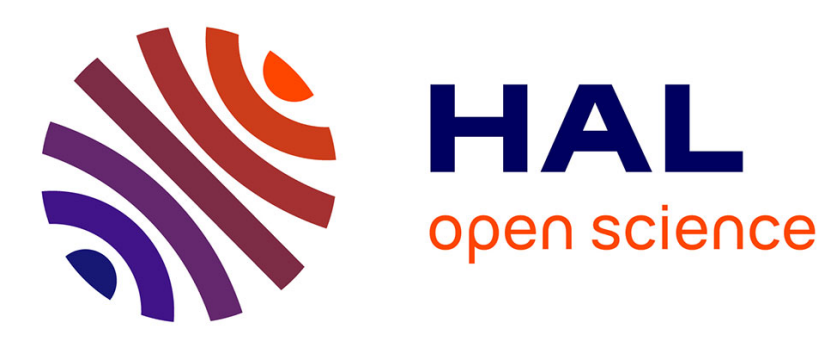

\title{
Power Control for Coordinated NOMA Downlink with Cell-Edge Users
}

\author{
Qianyun Guo, Chi Wan Sung, Yi Chen, Chung Shue Chen
}

\section{To cite this version:}

Qianyun Guo, Chi Wan Sung, Yi Chen, Chung Shue Chen. Power Control for Coordinated NOMA Downlink with Cell-Edge Users. IEEE Wireless Communications and Networking Conference (WCNC), Apr 2018, Barcelona, Spain. hal-01706276

\section{HAL Id: hal-01706276 \\ https://hal.inria.fr/hal-01706276}

Submitted on 11 Feb 2018

HAL is a multi-disciplinary open access archive for the deposit and dissemination of scientific research documents, whether they are published or not. The documents may come from teaching and research institutions in France or abroad, or from public or private research centers.
L'archive ouverte pluridisciplinaire HAL, est destinée au dépôt et à la diffusion de documents scientifiques de niveau recherche, publiés ou non, émanant des établissements d'enseignement et de recherche français ou étrangers, des laboratoires publics ou privés. 


\title{
Power Control for Coordinated NOMA Downlink with Cell-Edge Users
}

\author{
Qianyun Guo*, Chi Wan Sung*, Yi Chen ${ }^{\dagger}$ and Chung Shue Chen ${ }^{\ddagger}$ \\ *Department of Electronic Engineering, City University of Hong Kong, Kowloon, Hong Kong \\ ${ }^{\dagger}$ School of Science and Engineering, The Chinese University of Hong Kong, Shenzhen, China \\ ${ }^{\ddagger}$ Bell Labs, Nokia Paris-Saclay, 91620 Nozay, France \\ Email: qianyun.guo@telecom-bretagne.eu, albert.sung@cityu.edu.hk, \\ yichen@cuhk.edu.cn, chung_shue.chen@nokia-bell-labs.com
}

\begin{abstract}
Non-orthogonal multiple access (NOMA) is an effective means to improve the spectral efficiency of a wireless communication system. When applied to cellular networks, cell edge users may suffer from low bit rate, or the associated base stations may need to use excessively high power to serve those users. In order to alleviate the problem, this paper considers the integration of NOMA with coordinated transmission techniques. A two-cell system is considered, in which there are two users near their associated base stations and a cell edge user served by both base stations. It is assumed that each user has a data rate requirement, and the system objective is to minimize the total transmit power. With a formal problem formulation, the feasibility of the problem is characterized by using Helly's theorem. When the problem is feasible, we design both centralized and distributed algorithms to solve it. Numerical results show that NOMA can significantly outperform an orthogonal multiple access scheme in terms of power consumption and outage probability.

Index Terms-Non-orthogonal multiple access (NOMA), coordinated multipoint (CoMP), power control, power domain multiplexing.
\end{abstract}

\section{INTRODUCTION}

To satisfy the growing demand of mobile services, high requirements have been requested for future generations of mobile celluar systems. Among them, high spectral efficiency brings a big challenge to the multiple access scheme. Due to the scarcity of available radio spectrum, how to do it in an efficient way appears particularly important. To allocate limited spectral resources to multiple users, conventional schemes are adopted, which focus on time, code or frequency domains (TDMA, CDMA, OFDMA, etc.). These schemes can avoid the mutual interference between users by orthogonal design in the corresponding domain. However, the potential of spectral resources are not fully exploited by these schemes and they are not optimal in the field of spectral efficiency. In this case, non-orthogonal multiple access (NOMA) is proposed to provide higher spectral efficiency [1]. The prevalent concept of NOMA is power domain multiplexing. Different transmit powers are assigned to different users according to their channel conditions. For downlink, transmitters broadcast the messages of several users with superposition coding in the

This work was partially supported by a grant from the Research Grants Council of the Hong Kong Special Administrative Region, China (Project No. CityU 121713). same time and frequency block and users can decode their own message using successive interference cancellation (SIC).

Since 2013, NOMA has attracted more and more attention. In [2] [3], the authors adopted suboptimal fractional transmit power control (FTPC) to the NOMA system, which is similar to the power allocation in LTE and compared the sum rate performance of the NOMA system with the OMA system. A distributed optimal solution of downlink multiple-user system was proposed in [4] and it is showed that NOMA can save more energy than OMA.

However, due to the decoding order of SIC, equal performance can be difficult to achieve for the users near to BSs and the cell-edge users which have worse channel conditions. In [5], [6], the near-BSs users are used as relays to construct a cooperative NOMA system. The integration of NOMA system with beamforming technique was adopted in [7] [8] to improve user fairness. These methods need extra complex technique while the coordinated NOMA (CO-NOMA) can guarantee weak users' performance in a simpler way. In [9], with the assumption of the symmetric channel conditions and equal transmit power of near-BSs users, Alamouti code was used by two coordinated BSs and the performance in terms of the ergodic capacity was discussed. The work in [10] evaluated the system-level performance of CO-NOMA system in which all users are served in a coordinated way with a suboptimal power allocation solution of FTPA. Besides, a multi-tier NOMA strategy was considered in [11].

Different from [9]-[11], which focus on the sum rate performance, in this paper, we concern more about power consumption of a CO-NOMA system. The coordinated transmission of two BSs is adopted to improve the performance of a cell-edge user. We formulate the problem with a optimization parameter and show a necessary and sufficient condition of the feasibility derived from Helly's theorem [12]. Next, we propose a centralized optimal solution and a distributed suboptimal solution aiming to different scenarios. Finally, we simulate different scenarios of CO-NOMA as well as optimal solution of OMA and compare their numerical results.

The organization of the rest of this paper is as follows. Section II describes the system model and problem setup. The feasibility of the problem are discussed in Section III. Section IV presents two proposed solutions and algorithms. 


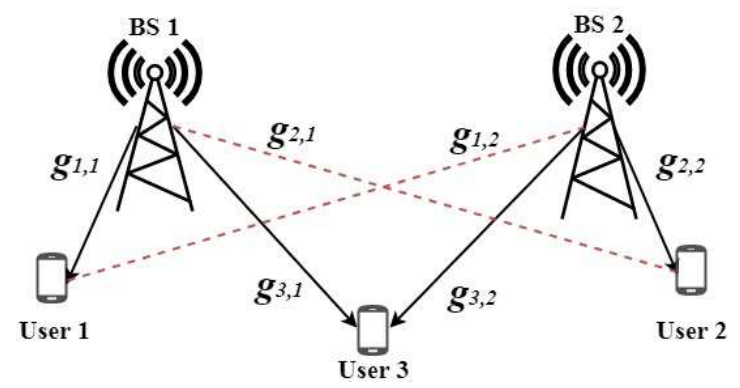

Fig. 1. Two-cell downlink NOMA system model with three users

Section $\mathrm{V}$ reports their performance and compares the optimal solution of OMA in terms of power consumption. Finally, the conclusion of this paper is showed in Section VI.

\section{SYSTEM MODEL}

We consider the downlink of a NOMA system with two base stations (BS) which use the same channel. As shown in Fig.1, each BS serves a user near to it (user 1 or user 2) while the cell-edge user (user 3 ) is served by both BSs. For $u \in\{1,2,3\}, b \in\{1,2\}$, let $g_{u, b}$ be the power gain of the link from BS $b$ to user $u$, and let $r_{u}$ be the rate requirement of user $u$. The bandwidth of the channel is $W$.

As user 3 is attached to two BSs, each BS broadcasts messages to its two users with superposition coding and the messages to user 3 from different BSs are the same. In other words, the transmitted signal of BS $b$ is $\sqrt{p_{b}} X_{b}+\sqrt{p_{3, b}} X_{3}$ for $b \in\{1,2\}$, where $p_{1}$ and $p_{2}$ are the powers for users 1 and 2 while $p_{3,1}$ and $p_{3,2}$ both are the powers for user 3 . So the total transmit power of $\mathrm{BS} 1$ is $p_{1}+p_{3,1}$ and the total transmit power of BS 2 is $p_{2}+p_{3,2}$. Let $\overrightarrow{\boldsymbol{p}}=\left(p_{1}, p_{2}, p_{3,1}, p_{3,2}\right)$. For $u \in\{1,2,3\}$, the received signal of user $u$ is

$$
\begin{aligned}
Y_{u}= & \sqrt{g_{u, 1} p_{1}} X_{1}+\sqrt{g_{u, 2} p_{2}} X_{2} \\
& +\left(\sqrt{g_{u, 1} p_{3,1}}+\sqrt{g_{u, 2} p_{u, 2}}\right) X_{3}+N_{u},
\end{aligned}
$$

where $N_{u}$ is a Gaussian random variable with variance $n_{u}>$ 0 .

For user 1, with the decodable constraint to be described later, it can first decode the message for user 3, use successive interference cancellation (SIC) to remove it from its received signal, and regard the signal from BS 2 as noise. It is similar for the other near-BS user (i.e., user 2). For the cell-edge user (i.e. user 3 ), the messages of users 1 and 2 are not decoded and the interfering signals are simply treated as noise. Let the noise power spectral density at each user be $N_{0}$. The postprocessing SINRs (i.e. after interference cancellation) of the three users can then be expressed as:

$$
\begin{gathered}
\Gamma_{1}=\frac{g_{1,1} p_{1}}{N_{0} W+g_{1,2}\left(p_{2}+p_{3,2}\right)}, \\
\Gamma_{2}=\frac{g_{2,2} p_{2}}{N_{0} W+g_{2,1}\left(p_{1}+p_{3,1}\right)},
\end{gathered}
$$

$$
\Gamma_{3}=\frac{\left(\sqrt{g_{3,1} p_{3,1}}+\sqrt{g_{3,2} p_{3,2}}\right)^{2}}{N_{0} W+g_{3,1} p_{1}+g_{3,2} p_{2}} .
$$

Note that we have assumed that the signals transmitted by both $\mathrm{BSs}$ for user 3 are aligned in phase so that the received power of the two signals can add up in. The corresponding rate of user $u$ is then given as

$$
R_{u}(\overrightarrow{\boldsymbol{p}})=W \log _{2}\left(1+\Gamma_{u}\right) .
$$

To make sure that users 1 and 2 can both decode the message for user 3 , the following constraints should be imposed:

$$
\begin{aligned}
& R_{1}^{\prime}=W \log _{2}\left(1+\frac{g_{1,1} p_{3,1}}{N_{0} W+g_{1,2}\left(p_{2}+p_{3,2}\right)+g_{1,1} p_{1}}\right) \geq r_{3} \\
& R_{2}^{\prime}=W \log _{2}\left(1+\frac{g_{2,2} p_{3,2}}{N_{0} W+g_{2,1}\left(p_{1}+p_{3,1}\right)+g_{2,2} p_{2}}\right) \geq r_{3}
\end{aligned}
$$

Although both BS 1 and 2 transmits the message of user 3, the signals arrived at users 1 or 2 cannot be aligned in phase due to different propagation delays. Therefore, in (5) we have assumed user 1 decodes the message of user 3 from the signal transmitted by BS 1, treating the signal transmitted by BS 2 as noise. The same assumption applies to (6).

The objective of this work is to minimize the sum of transmit power with the constraints of the minimum rate requirements and the decodable constraints. The problem can be formulated as follows:

$$
\min \|\vec{p}\|_{1}
$$

subject to

$$
\begin{gathered}
R_{u}(\overrightarrow{\boldsymbol{p}}) \geq r_{u}, \quad u=1,2,3, \\
R_{i}^{\prime}(\overrightarrow{\boldsymbol{p}}) \geq r_{3}, \quad i=1,2, \\
\overrightarrow{\boldsymbol{p}} \geq 0 .
\end{gathered}
$$

We call this problem $\boldsymbol{p}$-OPT.

\section{FEASIBILITY}

Due to the expression of $\Gamma_{3}$, the constraints of this problem are not all linear. On the other hand, it can be expressed as a parameterized family of linear programming problems. To see this, we define a parameter $\alpha$ as the ratio between $p_{3,2}$ and $p_{3,1}$, i.e., $p_{3,2}=\alpha p_{3,1}$. Define $\gamma_{u} \triangleq 2^{2 r_{u}}-1$ for $u=1,2,3$.

Lemma 1 -OPT is feasible only if

$$
g_{1,1} g_{2,2}>\gamma_{3}^{2} g_{1,2} g_{2,1},
$$

and $\alpha \in\left(\frac{\gamma_{3} g_{2,1}}{g_{2,2}}, \frac{g_{1,1}}{\gamma_{3} g_{1,2}}\right)$.

Proof: To ensure that (5) and (6) can be satisfied with non-negative power values, we must have, respectively,

$$
\begin{aligned}
& g_{1,1}-\gamma_{3} g_{1,2} \alpha>0 \\
& g_{2,2} \alpha-\gamma_{3} g_{2,1}>0
\end{aligned}
$$


These inequalities together imply (11) and the feasible range of $\alpha$.

Assume that the condition in Lemma 1 holds. With slight abuse of notation, we shorten $\vec{p}$ to a three-dimensional vector by removing the last component $p_{3,2}$, since it can be expressed as $\alpha p_{3,1}$. Then, the constraints (8) and (9) can be written in matrix form as

$$
A \vec{p} \geq \vec{n},
$$

where

$$
\begin{aligned}
& A \triangleq\left(\begin{array}{c}
a_{1}^{1} \\
a_{2}^{1} \\
a_{3}^{1} \\
a_{3}^{2} \\
a_{3}^{3}
\end{array}\right) \\
& \triangleq\left(\begin{array}{c}
1 \\
-\frac{\gamma_{2} g_{2,1}}{g_{2,2}} \\
-\frac{\gamma_{3} g_{3,1}}{\left(\sqrt{g_{3,1}}+\sqrt{g_{3,2} \alpha}\right)^{2}} \\
-\frac{\gamma_{3} g_{1,1}}{g_{1,1}-\gamma_{3} g_{1,2} \alpha} \\
-\frac{\gamma_{3} g_{2,1}}{g_{2,2} \alpha-\gamma_{3} g_{2,1}}
\end{array}\right. \\
& \begin{array}{c}
-\frac{\gamma_{1} g_{1,2}}{g_{1,1}} \\
1 \\
-\frac{\gamma_{3} g_{3,2}}{\left(\sqrt{g_{3,1}}+\sqrt{g_{3,2} \alpha}\right)^{2}} \\
-\frac{\gamma_{3} g_{1,2}}{g_{1,1}-\gamma_{3} g_{1,2} \alpha} \\
-\frac{\gamma_{3} g_{2,2}}{g_{2,2} \alpha-\gamma_{3} g_{2,1}}
\end{array} \\
& \left.\begin{array}{c}
-\frac{\gamma_{1} g_{1,2} \alpha}{g_{1,1}} \\
-\frac{\gamma_{2} g_{2,1}}{g_{2,2}} \\
1 \\
1 \\
1
\end{array}\right)
\end{aligned}
$$

and

$$
\overrightarrow{\boldsymbol{n}} \triangleq\left[\begin{array}{c}
n_{1}^{1} \\
n_{2}^{1} \\
n_{3}^{1} \\
n_{3}^{2} \\
n_{3}^{3}
\end{array}\right] \triangleq\left(\begin{array}{c}
\frac{\gamma_{1} N_{0} W}{g_{1}, 1} \\
\frac{\gamma_{2} N_{0} W}{g_{2,2}} \\
\frac{\gamma_{3} N_{0} W}{\left(\sqrt{g_{3,1}}+\sqrt{g_{3,2} \alpha}\right)^{2}} \\
\frac{\gamma_{3} N_{0} W}{g_{1,1}-\gamma_{3} g_{1,2} \alpha} \\
\frac{\gamma_{3} N_{0} W}{g_{2,2} \alpha-\gamma_{3} g_{2,1}}
\end{array}\right) .
$$

Define the set of square matrices

$$
\Omega \triangleq\left\{\left[\begin{array}{l}
a_{1}^{1} \\
a_{2}^{1} \\
a_{3}^{i}
\end{array}\right] \in \mathbb{R}^{3 \times 3}, \text { where } i=1,2,3\right\},
$$

which consists of three square matrices. For every $G \in \Omega$, the matrix $\boldsymbol{I}-\boldsymbol{G}$ is irreducible. Note that $\boldsymbol{G}$ can be regarded as a link gain matrix for a conventional power-controlled system, which consists of three co-channel point-to-point unicast system. For this reason, we call each $G$ a unicast setting. Note also that each $\boldsymbol{G}$ is parameterized by $\alpha$.

The following three results are needed for us to derive feasibility conditions of $\boldsymbol{p}$-OPT. The first one is a well-known result in the classical power control theory, the second one is a power control result originally derived for a multicast system, while the second one is a basic result in discrete geometry.

Theorem 1 (Power control theorem [13]-[15]) Consider a unicast setting $\boldsymbol{G}$ and assume $\boldsymbol{I}-\boldsymbol{G}$ is irreducible. The following statements are equivalent:

1) There exists a positive power vector $\overrightarrow{\boldsymbol{p}}$ such that $\boldsymbol{G} \overrightarrow{\boldsymbol{p}} \geq$ $\vec{n}$.

2) $\lambda(\boldsymbol{I}-\boldsymbol{G})<1$, where $\lambda(\boldsymbol{I}-\boldsymbol{G})$ is the Perron-Frobenious eigenvalue of $\boldsymbol{I}-\boldsymbol{G}$.

3) $\boldsymbol{G}^{-1}=\sum_{k=0}^{\infty}(\boldsymbol{I}-\boldsymbol{G})^{k} \geq \mathbf{0}$ exists.
Lemma 2 ( [16]) Consider $\tilde{\boldsymbol{G}}, \hat{\boldsymbol{G}} \in \Omega$ such that $\tilde{\boldsymbol{G}}$ differs from $\hat{\boldsymbol{G}}$ only in one row. Let $\tilde{\boldsymbol{p}}=\tilde{\boldsymbol{G}}^{-1} \overrightarrow{\boldsymbol{n}}_{\tilde{\boldsymbol{G}}}$ and $\hat{\boldsymbol{p}}=\hat{\boldsymbol{G}}^{-1} \overrightarrow{\boldsymbol{n}}_{\hat{\boldsymbol{G}}}$. There exists $\boldsymbol{p} \in\{\tilde{\boldsymbol{p}}, \hat{\boldsymbol{p}}\}$ such that $\tilde{\boldsymbol{G}} \boldsymbol{p} \geq \overrightarrow{\boldsymbol{n}}_{\tilde{\boldsymbol{G}}}$ and $\hat{\boldsymbol{G}} \boldsymbol{p} \geq \overrightarrow{\boldsymbol{n}}_{\hat{\boldsymbol{G}}}$.

Theorem 2 (Helly's theorem [12]) Let $F$ be a finite collection of convex sets in $\mathbb{R}^{N}$. The intersection of all the sets of $F$ is non-empty if and only if any $N+1$ of them has non-empty intersection.

Theorem 3 Given an instance of $\boldsymbol{p}$-OPT in which (11) holds, the instance is feasible if and only if there exists $\alpha \in$ $\left(\frac{\gamma_{3} g_{2,1}}{g_{2,2}}, \frac{g_{1,1}}{\gamma_{3} g_{1,2}}\right)$ such that

$$
\max _{\boldsymbol{G} \in \Omega} \lambda(\boldsymbol{I}-\boldsymbol{G})<1 .
$$

Proof: If the problem instance has a feasible solution, there exists a power vector $\overrightarrow{\boldsymbol{p}} \geq 0$ such that $\boldsymbol{A} \overrightarrow{\boldsymbol{p}} \geq \overrightarrow{\boldsymbol{n}}$. Then $\forall \boldsymbol{G} \in \Omega, \boldsymbol{G} \overrightarrow{\boldsymbol{p}} \geq \overrightarrow{\boldsymbol{n}} \geq 0$. According to the power control theorem, $\forall \boldsymbol{G} \in \Omega, \lambda(\boldsymbol{I}-\boldsymbol{G})<1$. So, $\max _{\boldsymbol{G} \in \Omega} \lambda(\boldsymbol{I}-\boldsymbol{G})<1$. The necessity is proved.

Next, assume $\max _{\boldsymbol{G} \in \Omega} \lambda(\boldsymbol{I}-\boldsymbol{G})<1$. By Theorem $1, \forall \boldsymbol{G} \in$ $\Omega$, there exists $\boldsymbol{G}^{-1} \geq 0$, and $\overrightarrow{\boldsymbol{p}}=\boldsymbol{G}^{-1} \overrightarrow{\boldsymbol{n}} \geq \mathbf{0}$ exists.

We first define a convex set $A_{1}^{1}=\left\{\overrightarrow{\boldsymbol{p}} \in \mathbb{R}^{3}: \boldsymbol{a}_{1}^{1} \overrightarrow{\boldsymbol{p}} \geq n_{1}^{1}\right\}$. Similarly, we can define the convex sets $A_{2}^{1}, A_{3}^{1}, A_{3}^{2}$, and $A_{3}^{3}$. Among these five convex sets, we choose any four of them and take the intersection. There are five possible combinations. We partition them into two groups, where the first group is $\left\{A_{1}^{1} \cap A_{2}^{1} \cap A_{3}^{1} \cap A_{3}^{2}\right\},\left\{A_{1}^{1} \cap A_{2}^{1} \cap A_{3}^{1} \cap A_{3}^{3}\right\}$ and $\left\{A_{1}^{1} \cap A_{2}^{1} \cap A_{3}^{2} \cap A_{3}^{3}\right\}$.

Let

$$
G_{1}=\left[\begin{array}{l}
a_{1}^{1} \\
a_{2}^{1} \\
a_{3}^{i}
\end{array}\right] \text { and } G_{2}=\left[\begin{array}{l}
a_{1}^{1} \\
a_{2}^{1} \\
a_{3}^{j}
\end{array}\right],
$$

where $i \neq j$. By Lemma 2, there exists $\overrightarrow{\boldsymbol{p}}$ such that $\boldsymbol{G}_{\mathbf{1}} \overrightarrow{\boldsymbol{p}} \geq$ $\overrightarrow{\boldsymbol{n}_{\mathbf{1}}}$ and $\boldsymbol{G}_{\mathbf{2}} \overrightarrow{\boldsymbol{p}} \geq \overrightarrow{\boldsymbol{n}_{\mathbf{2}}}$. So $\overrightarrow{\boldsymbol{p}} \in\left\{A_{1}^{1} \cap A_{2}^{1} \cap A_{3}^{i} \cap A_{3}^{j}\right\}$ and the intersection of each combination in this group is not empty.

Next, consider the second group, which consists of $\left\{A_{1}^{1} \cap A_{3}^{1} \cap A_{3}^{2} \cap A_{3}^{3}\right\}$ and $\left\{A_{2}^{1} \cap A_{3}^{1} \cap A_{3}^{2} \cap A_{3}^{3}\right\}$. We want to show that both of them are non-empty. By symmetry, it suffices to consider only $\left\{A_{1}^{1} \cap A_{3}^{1} \cap A_{3}^{2} \cap A_{3}^{3}\right\}$. We can first let $p_{2}=0$. The resulting system is equivalent to a system characterized by the $4 \times 2$ matrix $\boldsymbol{A}^{\prime}$ which is constructed by removing the second row and second column from $\boldsymbol{A}$. Similarly, we can define $\Omega^{\prime}$ and $G^{\prime}$ for $\boldsymbol{A}^{\prime}$. Note that the dimension of the power vector of this system is reduced from 3 to 2 . Now we want to prove that the new system has a feasible solution. We repeat the previous argument. For the new system, there are four combinations of any three convex sets. Like the original system, the first group is $\left\{A^{\prime 1} \cap A_{1}^{\prime 1} \cap A_{3}^{\prime 2}{ }_{3}\right\}$, $\left\{A^{\prime 1} \cap A_{1}^{\prime 1} \cap A_{3}^{\prime 3}{ }_{3}\right\}$ and $\left\{A^{\prime 1} \cap A_{1}^{\prime 2} \cap A_{3}^{\prime 3}{ }_{3}\right\}$. We can prove that each of them is non-empty in the same way with the first group of the original system with Lemma 1. The second group consists of only $\left\{A^{\prime 1} \cap{ }_{3}^{\prime 2} \cap{ }_{3}^{\prime} \cap A_{3}^{3}\right\}$, which is also nonempty, since we can easily find a feasible solution: $p_{1}^{\prime}=0$ and $p_{3,1}^{\prime}=\max \left\{n_{3}^{1}, n_{3}^{2}, n_{3}^{3}\right\}$. By Helly's theorem, there exists $\overrightarrow{\boldsymbol{p}^{\prime}}$ 
such that $\boldsymbol{A}^{\prime} \overrightarrow{\boldsymbol{p}^{\prime}} \geq \overrightarrow{\boldsymbol{n}^{\prime}}$. Then inserting $p_{2}=0$ back into $\overrightarrow{\boldsymbol{p}^{\prime}}$, we can get $\overrightarrow{\boldsymbol{p}} \in\left\{A_{1}^{1} \cap A_{3}^{1} \cap A_{3}^{2} \cap A_{3}^{3}\right\}$.

In conclusion, as the intersection of all combinations of any four convex sets are non-empty, by Helly's theorem, the intersection of all convex sets is non-empty and the problem is feasible. The sufficiency is proved.

We remark that our approach can be generalized to the case where there are maximum power constraints imposed at the base stations, based on the results in [17]. Moreover, our approach can also be extended to a large network which consists of multiple base stations and users.

\section{Power Control Algorithms}

In this section, we consider two approaches to solve $\boldsymbol{p}$-OPT. One is centralized, which requires a controller which knows all the channel gain information. This approach may not be practical but it serves a benchmark for comparison. The other one is a distributed approach, which removes the burden of measuring cross link gains, which is much more desirable from a practical viewpoint.

\section{A. Centralized Algorithm}

In the centralized scenario, we assume that there is a central controller which knows the values of all the channel gains. According to the constraint matrix, the solution can be decomposed into two steps:

1) Choose a value for the optimization parameter $\alpha$.

2) Given the value of $\alpha$, optimize the total power consumption.

In the first step, the controller performs one-dimensional grid search over $\alpha$ within the range $\left(\frac{\gamma_{3} g_{2,1}}{g_{2,2}}, \frac{g_{1,1}}{\gamma_{3} g_{1,2}}\right)$. Given each value of $\alpha$, the second step is a linear programming problem, which can be efficiently solved. It is clear that the solution obtained is optimal.

Alternatively, this problem can also be solved by convex optimization techniques. Note that in $\boldsymbol{p}$-OPT, all the constraints are linear except the one in (8) with $u=3$, i.e.,

$$
R_{3}(\overrightarrow{\boldsymbol{p}}) \geq r_{3} .
$$

We can rewrite it as

$$
\begin{array}{r}
g_{3,1}\left(p_{3,1}-\gamma_{3} p_{1}\right)+g_{3,2}\left(p_{3,2}-\gamma_{3} p_{2}\right)+2 \sqrt{g_{3,1} g_{3,2} p_{3,1} p_{3,2}} \\
\geq \gamma_{3} N_{0} W .
\end{array}
$$

Since $\sqrt{p_{3,1} p_{3,2}}$ is a concave function, the whole problem can be cast as a convex optimization problem, and thus can be solved by standard solvers.

\section{B. Distributed Algorithm}

In the distributed scenario, each BS knows only the link gains of the users associated with it, i.e., BS 1 knows the values of $g_{1,1}$ and $g_{3,1}$ while BS 2 knows the values of $g_{2,2}$ and $g_{3,2}$. The cross link gains, $g_{1,2}$ and $g_{2,1}$ are not known by the system. This consideration is especially important for a large network, since there are many interfering signals that are treated in noise during decoding. It is practically impossible to measure all the cross link gains in the network.
For this scenario, we propose a suboptimal solution. We adopt the same two-step approach in the previous subsection. The first step is to choose a value of $\alpha$ to approximate the optimal value of $\alpha$. Since cross-link gains have less influence in this system compared to other parameters, we neglect the cross-link gains, assuming that they are zero and solve for the optimal value of $\alpha$ for this simplified system. Note that without cross-link gains, the system becomes

$$
\boldsymbol{A} \overrightarrow{\boldsymbol{p}} \geq \overrightarrow{\boldsymbol{n}}
$$

where

$$
\boldsymbol{A}=\left(\begin{array}{ccc}
1 & 0 & 0 \\
0 & 1 & 0 \\
-\frac{\gamma_{3} g_{3,1}}{\left(\sqrt{g_{3,1}}+\sqrt{g_{3,2} \alpha}\right)^{2}} & -\frac{\gamma_{3} g_{3,2}}{\left(\sqrt{g_{3,1}}+\sqrt{g_{3,2} \alpha}\right)^{2}} & 1 \\
-\gamma_{3} & 0 & 1 \\
0 & -\frac{\gamma_{3}}{\alpha} & 1
\end{array}\right)
$$

and

$$
\overrightarrow{\boldsymbol{n}}=\left(\begin{array}{c}
\frac{\gamma_{1} N_{0} W}{g_{1}, 1} \\
\frac{\gamma_{2} N_{0} W}{g_{2,2}} \\
\frac{\gamma_{3} N_{0} W}{\left(\sqrt{g_{3,1}}+\sqrt{g_{3,2} \alpha}\right)^{2}} \\
\frac{\gamma_{3} N_{0} W}{g_{1}, 1} \\
\frac{\gamma_{3} N_{0} W}{g_{2,2} \alpha}
\end{array}\right)=\left[\begin{array}{c}
n_{1}^{1} \\
n_{2}^{1} \\
n_{3}^{1} \\
n_{3}^{2} \\
n_{3}^{3}
\end{array}\right]
$$

By the constraints above, the transmit power values to user 1 and user 2 are independent of the transmit power value to user 3 , since the interference of user 3 is canceled at user 1 and user 2. Obviously, the minimum transmit power values to user 1 and user 2 are $n_{1}^{1}$ and $n_{2}^{1}$, respectively. The remaining problem is to minimize $p_{3,1}$ and $p_{3,2}$ with the constraints as follows:

$$
\left\{\begin{array}{l}
p_{3,1} \geq \frac{\left.\gamma_{3} N_{0} W\left(1+\gamma_{1}\right)\right)}{g_{1,1}} \triangleq a, \\
p_{3,2} \geq \frac{\left.\gamma_{3} N_{0} W\left(1+\gamma_{2}\right)\right)}{g_{2,2}} \triangleq b, \\
\sqrt{g_{3,1} p_{3,1}}+\sqrt{g_{3,2} p_{3,2}} \geq c,
\end{array}\right.
$$

where $c \triangleq \sqrt{\gamma_{3}\left(N_{0} W+g_{3,1} n_{1}^{1}+g_{3,2} n_{2}^{1}\right)}$.

Let $x \triangleq \sqrt{p_{3,1}}$ and $y \triangleq \sqrt{p_{3,2}}$. We can then re-write the above problem as follows:

$$
\begin{gathered}
\min x^{2}+y^{2} \\
\text { s.t. }\left\{\begin{array}{c}
x \geq \sqrt{a} \\
y \geq \sqrt{b} \\
\sqrt{g_{3,1}} x+\sqrt{g_{3,2}} y \geq c
\end{array}\right.
\end{gathered}
$$

It can be seen that we need to find a feasible point that minimize its distance from the origin.

If we have $\sqrt{g_{3,1} a}+\sqrt{g_{3,2} b} \geq c$, then we can obtain $x=$ $\sqrt{a}, y=\sqrt{b}$, and $\alpha=b / a$. Otherwise, the optimal point must be on line $L$ defined by $\sqrt{g_{3,1}} x+\sqrt{g_{3,2}} y=c$. We assume that the distance between the origin and the point $\left(x_{0}, y_{0}\right)$ is the minimum among the distances of all the point on line $L$. Since the line connecting $\left(x_{0}, y_{0}\right)$ to the origin must be 
perpendicular to line $L$, we must have $y_{0} / x_{0}=\sqrt{g_{3,2} / g_{3,1}}$. Solving the two simultaneous equations, we obtain

$$
x_{0}=\frac{c \sqrt{g_{3,1}}}{g_{3,1}+g_{3,2}} \text { and } y_{0}=\frac{c \sqrt{g_{3,2}}}{g_{3,1}+g_{3,2}} .
$$

So for this case, the optimal point of $\left(p_{3,1}, p_{3,2}\right)$ can be expressed as follows:

$$
\left\{\begin{array}{ll}
\left(x_{0}^{2}, y_{0}^{2}\right) & \text { if } x_{0} \geq \sqrt{a} \text { and } y_{0} \geq \sqrt{b} \\
\left(a, \frac{\left(c-\sqrt{g_{3,1}}\right)^{2}}{g_{3,2}}\right) & \text { if } x_{0}<\sqrt{a} \text { and } y_{0} \geq \sqrt{b} \\
\left(\frac{\left(c-\sqrt{g_{3,2} b}\right)^{2}}{g_{3,1}}, b\right) & \text { if } x_{0} \geq \sqrt{a} \text { and } y_{0}<\sqrt{b}
\end{array} .\right.
$$

Hence, $\alpha$ can be obtained. With the estimated value of $\alpha$, we use an iterative distributed algorithm for the second step. The system in (17), with cross link gains non-zero, can be expressed as

$$
\vec{p} \geq \boldsymbol{I}(\vec{p}),
$$

where $I=\left[\begin{array}{lllll}I_{1}^{1} & I_{2}^{1} & I_{3}^{1} & I_{3}^{2} & I_{3}^{3}\end{array}\right]^{T}$ is called the interference function. It can be easily proved that $\boldsymbol{I}$ satisfies the following three properties and is thus called a standard interference function [18]:

1) Positivity: $\boldsymbol{I}(\overrightarrow{\boldsymbol{p}}) \geq 0$

2) Monotonicity: If $\overrightarrow{\boldsymbol{p}} \geq \overrightarrow{\boldsymbol{p}}^{\prime}$, then $\boldsymbol{I}(\overrightarrow{\boldsymbol{p}}) \geq \boldsymbol{I}(\overrightarrow{\boldsymbol{p}})$

3) Scalability: For all $\alpha>1$, then $\alpha \boldsymbol{I}(\overrightarrow{\boldsymbol{p}}) \geq \boldsymbol{I}(\alpha \overrightarrow{\boldsymbol{p}})$

For our case, the algorithm can be written in the form:

$$
\overrightarrow{\boldsymbol{p}}^{(t)}=\boldsymbol{I}^{\prime}\left(\overrightarrow{\boldsymbol{p}}^{(t-1)}\right),
$$

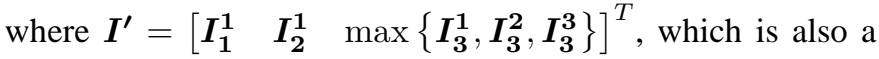
standard interference function by [18, Theorem 5]. In addition, the convergence of this algorithm has been proved in [18].

In this scenario, the two BSs do not need to know all channel information and they only have to communicate with each other in the first step. The search of optimal $\alpha$ is avoided by an estimated value. We will discuss the performance of these two algorithms in the next section.

\section{Simulation AND COMPARISON}

To evaluate the performance of our model, we first think about the downlink of a corresponding OMA system with two channels whose bandwidth are $w$ and $W-w$.The messages from BS 1 to user 1 and from BS 2 to user 2 share a common channel and the messages from BS 1 and BS 2 to user 3 use the other channel. Here, the rate constraints become:

$$
\begin{gathered}
R_{O M A 1}=w \log _{2}\left(1+\frac{g_{1,1} p_{1}}{N_{0} w+g_{1,2} p_{2}}\right) \geq r_{1} \\
R_{O M A 2}=w \log _{2}\left(1+\frac{g_{2,2} p_{2}}{N_{0} w+g_{2,1} p_{1}}\right) \geq r_{2} \\
R_{O M A 3}=(W-w) \log _{2}\left(1+\frac{\left(\sqrt{g_{3,1} p_{3,1}}+\sqrt{g_{3,2} p_{3,2}}\right)^{2}}{N_{0}(W-w)}\right) \\
\geq r_{3}
\end{gathered}
$$

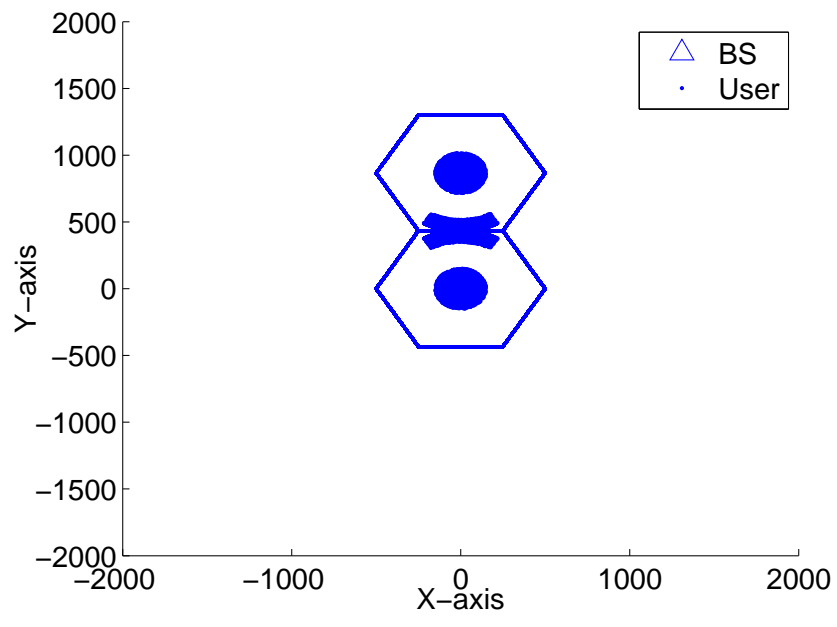

Fig. 2. Locations of the three users after 5000 times' simulation.

where $r_{u}$, for $u=1,2,3$, are the same rate requirements as for the NOMA schemes and the variable $w \in[0, W]$.

Given the value of $w$, it is straightforward to minimize the total power $\|\vec{p}\|_{1}$. Therefore, the optimal sum power for the OMA scheme can be obtained by a one-dimensional grid search for $w$.

Next, we compare the performances of OMA and NOMA systems. We consider two neighboring hexagonal cells with radius of 500 meters. As shown in Fig. 2, in each cell, there is one BS in the center and one user uniformly distributed in a circular area around BS. The radius of the circular area is 150 meters. And there is also one user located in the cell edge, the distance from this user to BS1 or BS2 can range from 350 meters to 650 meters. The system bandwidth is assumed to be $10 \mathrm{MHz}$ and the noise power spectral density is assumed to be $-174 \mathrm{dBm} / \mathrm{Hz}$. In the propagation model, we consider the distance-dependent path loss, shadow fading and small-scale fading. The path loss component is given by $128.1+37.6 \log _{10} d$, in which $d$ is the distance between the transmitter and the receiver in $\mathrm{km}$. Lognormal shadowing has the standard deviation of $8 \mathrm{~dB}$. For small-scale fading, each user experiences independent Rayleigh fading with variance 1 .

Fig. 3 shows the total power consumption of the two NOMA schemes and the OMA scheme with increasing rate requirement. We generate randomly 5,000 problem instances for each target rate. Among them, we calculate the average power consumption of the instance in which all the three schemes are feasible. We can observe both NOMA schemes outperform the OMA scheme and the advantage increases with the rate requirement. When the rate requirement is $12 \mathrm{Mbit} / \mathrm{s}$ for each user, the optimal NOMAscheme saves $37.52 \%$ of average power consumption and the distributed NOMA scheme saves $31.32 \%$ of average power consumption compared with the OMA scheme. Moreover, according to the performance, the distributed scheme can be a good approximation to the optimal centralized scheme, especially for low rate requirements. 


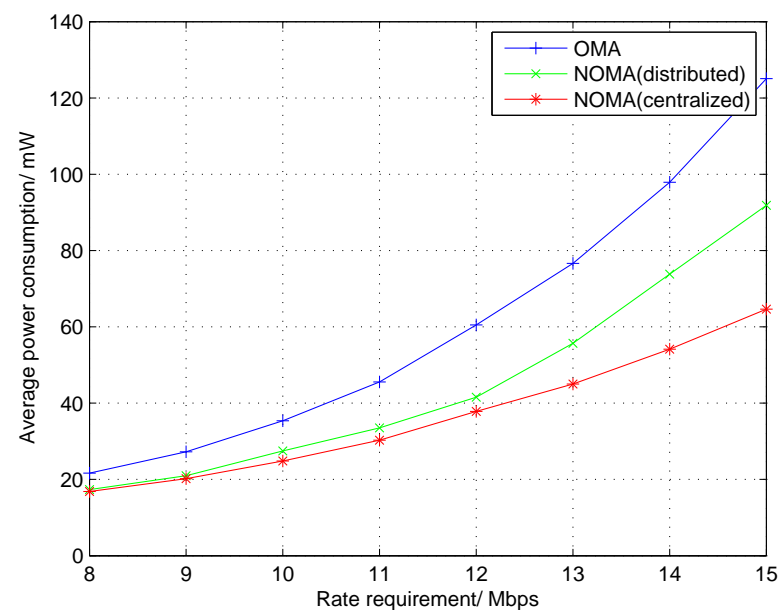

Fig. 3. Power consumption versus minimum rate requirements of three schemes.

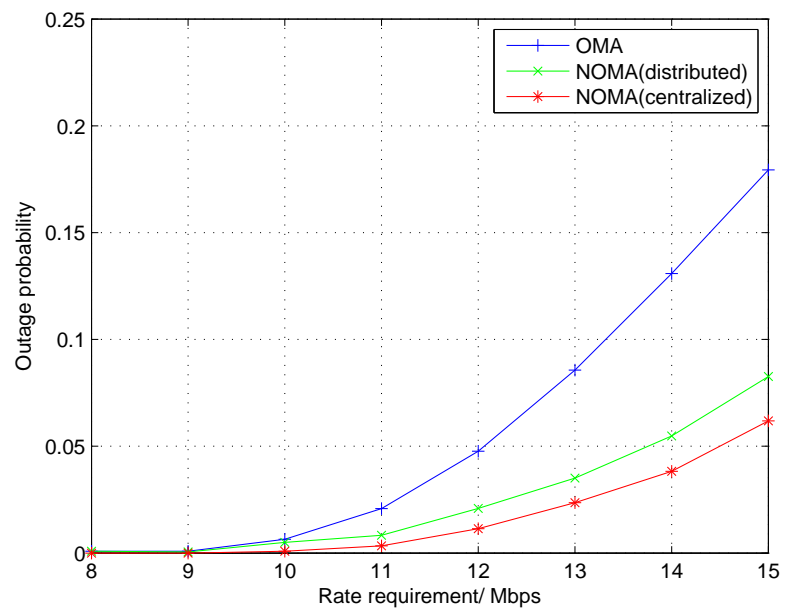

Fig. 4. Outage probability versus minimum rate requirements of three schemes.

To compare the outage probability of the three schemes, we assume the maximum transmit power of each base station is $100 \mathrm{~mW}$. Fig. 4 represents the numerical results of the outage probability, which is determined by the percentage of the outage instances among all the instances. With the growing rate requirements, the outage probabilities also increase and the NOMA schemes also outperform the OMA scheme significantly.

\section{CONCLUSION}

This work considers the downlink of a coordinated NOMA two-cell system, which serves two near-BS users and a celledge user. The power minimization problem for such a system is formulated subject to rate constraints. The feasibility issue is investigated, and a necessary and sufficient condition is derived by means of Helly's theorem. Furthermore, the power minimization problem is shown to be a parameterized family of linear programming problems, so that it can be solved by doing a grid search over a single parameter. Such an approach, however, requires the knowledge of all the link gains in the system, which is practically difficult to implement. For this reason, a distributed heuristic algorithm is proposed, which eliminates the need of measuring cross link gains. Numerical results show that it outperforms an OMA benchmark system.

While this work considers only a small network, the approach is expected to be applicable to a large network with many base stations and users. In a large network, however, some other issues need to be addressed, such as user grouping and base station association, which are left as future work.

\section{REFERENCES}

[1] Y. Saito, Y. Kishiyama, A. Benjebbour, T. Nakamura, A. Li, and K. Higuchi, "Non-orthogonal multiple access (noma) for future radio access," Proc. of IEEE VTC spring 2013, pp. 1-5, Jun. 2013.

[2] Y. Saito, A. Benjebbour, Y. Kishiyama, and T. Nakamura, "System level performance evaluation of downlink non-orthogonal multiple access (noma)," Proc. IEEE Annu. Symp. Personal Indoor and Mobile Radio Communications (PIMRC), pp. 611-615, Sep. 2013.

[3] A. Benjebbour, A. Li, Y. Saito, Y. Kishiyama, A. Harada, and T. Nakamura, "System-level performance of downlink NOMA for future lte enhancements," Proc. IEEE GLOBECOM, pp. 66-70, Dec. 2013.

[4] Y. Fu, Y. Chen, and C. W. Sung, "Distributed downlink power control for the non-orthogonal multiple access system with two interfering cells," Proc. IEEE International Conference on Communications, May 2016.

[5] Z. Ding, M. Peng, and H. V. Poor, "Cooperative non-orthogonal multiple access in 5 G systems," IEEE Commun. Lett., vol. 19, no. 8, pp. 14621465, Aug. 2015.

[6] J. Men and J. Ge, "Non-orthogonal multiple access for multiple antenna relaying networks," IEEE Commun. Lett., vol. 19, no. 10, p. 16861689 , Oct. 2015

[7] B. Kim, S. Lim, H. Kim, S. Suh, J. Kwun, S. Choi, C. Lee, and S. L. an D. Hong, "Non-orthogonal multiple access in a downlink multiuser beamforming system," Proc. IEEE Milit. Commun. Conf., pp. 1278 1283, Nov. 2013

[8] K. Higuchi and Y. Kishiyama, "Non-orthogonal access with random beamforming and intra-beam sic for cellular MIMO downlink," Proc. IEEE Veh. Technol. Conf., pp. 1-5, Sep. 2013.

[9] J. Choi, "Non-orthogonal multiple access in downlink coordinated twopoint systems," IEEE Commun. Lett., vol. 18, no. 2, pp. 313-316, Feb. 2014.

[10] A. Beylerian and T. Ohtsuki, "Coordinated non-orthogonal multiple access (CO-NOMA)," Proc. IEEE GLOBECOM, Dec. 2016.

[11] Y. Tian, A. R. Nix, and M. Beach, "On the performance of multi-tier NOMA strategy in coordinated multi-point networks," IEEE Commun. Lett., to appear.

[12] B. Alexander, "A course in convexity," American Mathematical Society, vol. 54, 2002.

[13] J. Zander, "Performance of optimum transmitter power control in cellular radio systems," IEEE Transactions on Vehicular Technology, vol. 41, pp. 57-62, Feb. 1992.

[14] G. J. Foschini and Z. Miljanic, "A simple distributed autonomous power control algorithm and its convergence," IEEE Transactions on Vehicular Technology, vol. 42, pp. 641-646, Nov. 1993.

[15] N. Bambos, S. C. Chen, and G. J. Pottie, "Channel access algorithms with active link protection for wireless communication networks with power control," IEEE/ACM Transactions on Networking, vol. 8, no. 5, pp. 583-597, Oct. 2000.

[16] Y. Chen and C. W. Sung, "Characterization of SINR region for multiple interfering multicast in power-controlled systems," arXiv preprint arXiv:1505.06882, May 2015.

[17] H. Mahdavi-Doost, M. Ebrahimi, and A. Khandani, "Characterization of SINR region for interfering links with constrained power," IEEE Trans. Inform. Theory, vol. 56, no. 6, 2010.

[18] R. D. Yates, "A framework for uplink power control in cellular radio systems," IEEE J. Select. Areas Commun., vol. 13, no. 7, pp. 1341-1347, Sep. 1995. 